\title{
4 EPHEMERIDES (EPHEMERIDES)
}

President: P. K. Seidelmann

Vice President: B. D. Yallop

Organizing Committee: V. K. Abalakin, J. Chapront, R. L. Duncombe, H. Kinoshita, Y. Kubo, J. H. Lieske, B. Morando, H. Schwan, Fu Tong.

\section{Introduction}

This report covers the period from 1 July 1987 to 30 June 1990. The ephemerides that have been published during this period have made use of the system of astronomical constants adopted at the $16 \mathrm{th}$ General Assembly of the IAU in Grenoble. At the same time that the use of the FK5 system on the J2000 epoch is increasing and catalogues are becoming available for that system, there is an IAU Working Group on Reference Systems with subgroups on astronomical constants, nutation of the Earth, time and reference frames/origin. The purpose is to define a future reference system and resolve some of the difficulties that occur in the system. It is anticipated that this Working Group will develop recommendations for consideration at the 21 st General Assembly of the IAU.

\section{International and National Ephemerides}

1. The Fundamental System

The computation and publication of Apparent Places of Fundamental Stars (APFS) has been continued at Astronomisches Rechen-Institut at Heidelberg, F. R. Germany. Starting with the volume for the year 1988 the APFS are based on the mean positions and proper motions as given in the EK5, Part 1 ("Basic FK5") which has been published in 1988 (Veroffentlighungen Astronomische Rechen-Institut, No. 32). The number of copies of the APFS has been reduced to 1000 .

\section{Ephemerides and Astronomical Data}

The Japanese Ephemeris (JE), the Nautical Almanac (NA) and the Abridged Nautical Almanac (ANA) have continued to be published for the years 1989, 1990 and 1991 by the Hydrographic Department of Japan (JHD), Tokyo. No major change in the contents has been made after the volume for 1985, in which was introduced a new scheme of computation based on the fundamental reference frame of FK5, numerical integration for the 
coordinates of the bodies in the solar system, day numbers in rectangular coordinates and so on. JE for the years 1985 onwards contain the explanation of the method on which the new series of JE are based.

JE for 1990 onwards contains Chebychev coefficients for the Moon's coordinates as a supplement and NA for 1989 onwards includes a supplement containing the coefficients for all the objects which constitute the main body of NA.

The Polaris Almanac for Azimuth determination, the Altitude and Azimuth Observation Almanac for Antarctic Observation and the Abstract from JE also have been compiled annually by JHD.

During the period under review the Astronomical Yearbook of the USSR for the years 1990, 1991 and 1992 have been published by the Institute for Theoretical Astronomy, (ITA), Acad. Sci. USSR, Leningrad. The fundamental ephemerides of the Sun, Moon and major planets were computed on the basis of DE200/LE200. Beginning from the issue for 1990 the Basic part of Fifth Fundamental Catalogue, and from 1992 - its Bright Extension, were introduced into the practice of calculation of the Mean and Apparent Places of the stars. Beginning from 1992 the expressions for the visual magnitudes for major planets, taken from G. DeVaucouleur's review (A. Dollfus. Surfaces and interiors of planets and satellites, 1970) are in use. The first version of the Astronomical Yearbook of the USSR on a floppy disk is now in preparation.

In 1988-1990 the issues of the Marine Astronomical Yearbook and Air Astronomical Yearbook for the years 1989, 1990 and 1991 were prepared by ITA, as well as one issue of Astronavigation Almanac for the years 1991 - 1995.

The Department of Almanac of the Purple Mountain Observatory computes the Chinese Astronomical Almanac, the Chinese Nautical Almanac and the Almanac for Land Surveyors every year.

Her Majesty's Nautical Almanac Office, Royal Greenwich Observatory, (HMNAO) and the Nautical Almanac Office, U S Naval Observatory (USNAO) have continued their cooperative preparation and publication of The Astronomical Almanac, The Nautical Almanac, The Air Almanac, and Astronomical Phenomena.

The Floppy Almanac, providing the basic astronomical data of the Astronomical Almanac, is available from USNAO for each year through 1999. The Satellite Almanac, a floppy disk providing the satellite positions for a period of approximately 10 years, is also available from USNAO.

Bureau des Longitudes, Paris, France (BdL) have published yearly the Connaissance des Temps, the Ephemerides Astronomiques (Annuaire du Bureau des Longitudes) and the Ephemerides Nautiques.

3. Special Publications

Some special publications which merit attention include 
"Compact Data for Navigation and Astronomy, 1991 - 1995" by B. D. Yallop and C. Y. Hohenkerk, published by Cambridge University Press and "Equation of Time" by D. Hughes, B. D. Yallop and C. Y. Hohenkerk, published in 1989 Mon. Not. R.A.S. pages 1529 1535. Two technical notes from the RGO, \#66 by C. Y. Hohenkerk, "Topps, a System for Printing Tables" and \#67 by B. D. Yallop and C. Y. Hohenkerk, "Astronomical Algorithms for Use with Microcomputers."

$U$ S Naval Observatory Circulars published during this triennium include \#171, "Computer Programs for Sun and Moon Illuminance with Contingent Tables and Diagrams" by $P$. M. Janiczek and J. A. DeYoung; \#172, "Total Solar Eclipse of 17 18 March 1988" by A. D. Fiala, J. A. Bangert and W. T. Harris; \#173 "Total Solar Eclipse of 2 July 1990" by Fiala, Bangert and Harris; \#174, "Total Solar Eclipse of 11 July 1991" by Bangert, Fiala and Harris and \#175 "Annular Solar Eclipse of 15 - 16 January 1991: by Bangert, Fiala and Harris.

A series of three papers appeared in the Astronomical Journal in January and April 1989 on the Mean and Apparent Place Computations in the New IAU system. The first two papers dealt with the transformation of catalogue systems to the $\mathrm{J} 2000$ system and the last paper with the Apparent Topocentric and Astrometric places of Planets and Stars.

USNAO has prepared a revised version of the Explanatory Supplement. Chapters and sections of this publication have been prepared by HMNAO, Jet Propulsion Laboratory and BdL. The publication should be available in 1991.

Many kinds of ephemerides were prepared in these years, e.g. the astronomical data for all calendars being published in USSR, "The ephemerides for comparison and positioning of the instruments" for the optical observations of the Sun, Moon, and planets at the astronomical observatories of the USSR, and the ephemerides for the observations of the different radio-sources with radio-telescope RATAN-600 (Zelenchukscaya, R.S.F.S.R.).

BdL published Three Supplements to Connaissance des Temps: Ephemerides of the Satellites of Jupiter, Saturn and Uranus that give the differential coordinates of the satellites and tables for the computation of the phenomena of the Galilean satellites - Phenomena and configurations of the Galilean satellites of Jupiter - configurations of the first eight satellites of Saturn. Two main improvements will be introduced in the Supplements to Connaissance des Temps from 1991: Ephemerides of the Martian moons and predictions of mutual phenomena for the Galilean satellites.

BdL have also produced the following ephemerides published in the Notes Scientifiques et Techniques du Bureau des Longitudes: Ephemerides de petites planetes de 1989 a 1991 (S018, S022, S023) - Determination d'orbites de cometes de 1989 a 1991 (S019, S020, S027) - ELP2000/85: une solution au mouvement de la Lune couvrant la periode historique (S021) Construction d'une ephemeride de phobos issue de la theorie 
ESAPHO (S024) - Modelisation des phenomenes mutuels (S030).

An Infrared point Source Catalog of 15560 sources was prepared by Yang, Xian, He and Yao at Purple Mountain observatory.

\section{Bases of the Ephemerides and Astronomical Data}

H. Schwan reports that work on the second part of the FK5 (The FK5 Extension) which is a joint project by ARI and USNO is near completion. More information on the status of the FK5 Extension, which will extend the fundamental system to about magnitude 9.5, is given in the report for Commission 8.

J. G. Williams, $X X$ Newhall, and J. O. Dickey report that three observatories are ranging the moon regularly. The last few years of data from the three sites can be fit with $3 \mathrm{~cm}$ scatter. The two decades of lunar laser ranging (LLR) data exceed an $18.6 \mathrm{yr}$. nodal period permitting the mutual orientation of the ecliptic, equator, and lunar orbit planes to be determined to better than two milliarcseconds and the precession constant to be separated from the principal nutation term. The lunar orbit and lunar physical librations are well determined by the observations and are computed from a simultaneous numerical integration. The mass ratio Sun/(Earth+Moon) is determined to within 0.003 . The lunar orbit was accurate enough to permit measuring the relativistic precession to within $2 \%$. The lunar and planetary ranging data can be fit simultaneously.

According to E. Myles Standish, Jr., JPL continues to improve its planetary ephemerides. Since the creation of DE200, a number of observations have been obtained, including both additional standard data (optical transits, radar and spacecraft ranging and lunar laser ranging), as well as a number of newer and more accurate data types (spacecraft tracking files, radio astrometry, photoelectric transits, astrolabe measurements, ring and disk occultations, and improved lunar laser ranging). The improvements since the creation of DE200 are noticeable, especially in the ephemerides of the outer planets. Knowledge from the voyager fly-bys of the four Jovian planets and from the mutual occultations of Pluto and Charon have enabled the full set of outer planet masses to be determined, thereby eliminating them as sources of orbital uncertainties. The remaining mass uncertainties are primarily those of the asteroids. The forces of Ceres, Pallas and Vesta are modeled separately; in addition, the forces of the outer 300 most important asteroids are modeled using best estimates of their masses, computed from their diameters and taxonomic classes. A new set of ephemerides, completely reduced and integrated in the J2000 reference system, is being created. This will incorporate the full data set, the latest improvements to the data reduction processes and the latest refinements to the gravitational equations of motion. 
R. A. Jacobson and J. H. Lieske report that JPI has developed ephemerides for most of the major natural planetary satellites in support of planetary exploration missions. Martian satellite ephemerides were prepared for the soviet Phobos mission from ground based and spacecraft data. The ephemerides were updated from observations by the soviet spacecraft. Galilean satellite ephemerides have been updated to "E3" in support of the Galileo project. For the voyager project, ephemerides of the six inner Saturnian sateliites, the five large Uranian satellites and the two Neptunian satellites were prepared. Improved ephemerides based on encounter observations have been prepared for the Saturn satellites. Ephemerides for the nine major Saturnian satellites will be improved for the Hubble Space Telescope and the Cassini mission.

A new basis for the ephemeris calculation is now being set up in ITA (Belikov M. V., Boyko V. N., Glebova N. I., Eroshkin G. I., Rumyantseva L. I., Sveshnikov M. L., Sveshnikova E. S., Trubitsyna A. A., Fursenko M. A., Chunaeva L. I., Shiryaev A. A. "The main stages of the construction of AE89 - the numerical ephemerides of the planets and Moon" - IAU Symposium No. 141 "INERTIAL COORDINATE SYSTEM ON THE SKY" Leningrad, October 1989). The problems solved or in the process of solution are as follows:

1) The refinement of the dynamically consistent numerical model of the orbital motion of 9 major planets, the five most massive asteroids and orbital-rotational motion of the Moon;

2) The construction of a data base of astrometric observations of the Sun, Moon, and planets (optical, radar, laser);

3) The determination of the dynamical parameters of the Solar system from the observations.

A standardized set of transportable computer software, developed on a building-block basis, to provide a computer based almanac is being developed at the USNAO. It is being developed with interfaces to a number of different computers, but with the expectation that the building blocks can be incorporated into other customized software.

The theory and observational data for the satellites of Saturn have been investigated by B. D. Taylor, A. T. Sinclair, P. J. Message, D. Harper, S. Kaimian and P. R. Strugnell. This work resulted in a series of papers published in Astronomy and Astrophysics. Astrometric observations of Neptune and Triton were obtained and compared with theory by D. B. Taylor, H.P. Jones, L. V. Morrison, C. A. Murray and I. P. Williams, and published in Astronomy and Astrophysics.

IV. Observational Data for Improving the Ephemerides

The services of the International Lunar Occultation Center have been continued since 1981. The number of the timing data collected at the Center was 38,644 from 38 countries during the 
years 1987 to 1989. Reports containing all the reduced data as well as the station coordinates are published annually. Nos. 6 to 8 were published in the period.

The software for the prediction of the lunar occultations as well as for the treatment of the observations became operational in ITA.

USNAO continued to mail occultation predictions to observers on an annual basis. Photographic observations of the larger satellites of Mars, Jupiter and Saturn were continued at USNAO by Pascu. Charge-coupled device observations of the faint satellites of Jupiter, Saturn, Uranus and Neptune continued by Seidelmann and Pascu using the 61 inch astrometric telescope in Flagstaff, Arizona.

During the "Phobos" campaign of the 1988 Mars opposition, M. Sveshnikov took part in observations of the Martian satellites with lunar-planetary menisk telescope (Ordubad Station of Pulkova Observatory. Azerbaidjan). He has made 63 photographic observations of Phobos and 54 of Deimos.

The problem of regularly obtaining new observations of the Solar system bodies, so urgent for our work, is about to be solved. Now ITA will carry out the coordination of such observations at USSR observatories in frames of the projects "Jupiter" and "Ceres." One of the products of this activity would be the construction of the data bases of observations for personal computers.

For HIPPARCOs mission BdL have established ephemerides of 60 minor planets for the input catalog, as well as ephemerides for Europa and Titan.

\section{Organizational Changes}

B. D. Yallop delineated the changes at HMNAO. The move of the RGO to Cambridge took place at the end of March 1990. HMNAO transferred with the observatory to a new building built on a site next to the Institute of Astronomy, Cambridge. The last superintendent of HMNAO, Dr. G. A. Wilkins, retired on 4 July 1989 after 38 years of service in HMNAO. Dr. B. D. Yallop has taken over as head of HMNAO.

The work of HMNAO was paid for by annual grants from the Science and Engineering Research Council and Ministry of Defense until the end of 1989 when it was changed to a repayment basis. This has involved major changes in the methods of working, for example, publications are produced in-house and methods are more automated to reduce checking and cut costs. The office now recovers the money from the sales of the almanacs, charges copyright fees for supplying data to other publishers, and charges for the supply of data to users, including the data for observatories. The income is sufficient to run the office. Any extra income may be used to increase staff or services. 